Egyptian Journal of Aquatic Biology and Fisheries

Zoology Department, Faculty of Science,

Ain Shams University, Cairo, Egypt.

ISSN $1110-6131$

Vol. 25(4): 587 - 601 (2021)

www.ejabf.journals.ekb.eg

\title{
Long-Term Toxic Effect of Inhaling Smokes of Cigarette and Incense on different stages of Culex pipiens
}

\author{
Khaled S. M. Osman, Mohammed Z. Y. Aly, Mervat A. B. Mahmoud* \\ Zoology Department, Faculty of Science, South Valley University, Qena, Egypt \\ "Corresponding Author: Mervat.mahmoud@sci.svu.edu.eg
}

\begin{abstract}
ARTICLE INFO
Article History:

Received: Aug. 4, 2021

Accepted: Aug.19, 2021

Online: Aug. 30, 2021

Keywords:

Toxicity, mosquitoes,

Culex pipiens,

cigarette,

mortality,

GC-MS

ABSTRACT

The toxic effects of inhaling cigarette and industrial incense smoke on the different stages of the aquatic insect Culex pipiens (L.) for two generations had been investigated. Eggs, larvae, pupae, and adult stages were exposed to the smoke daily for 82 days. The results showed that in the $1^{\text {st }}$ generation, cigarette smoke affected the mortality rate of larvae $(29.80 \%)$ and adults $(57.95 \%)$, while industrial incense smoke recorded a loss rate on the larvae $(47.56 \%)$ and to the adults $(32.61 \%)$. In the $2^{\text {nd }}$ generation, the highest loss rate was recorded for adult mortality $(75.68 \%)$ from cigarette smoke. Larval and adult mortality was affected by industrial incense smoke by $86.67 \%$ and $54.55 \%$, respectively. Moreover, egg production of the $1^{\text {st }}$ to $2^{\text {nd }}$ generations was affected negatively by cigarettes $(88.06 \%)$ and positively by industrial incense $(36.99 \%)$. X-ray fluorescence spectrometer analysis showed incense had a major amount of calcium $(78.24 \%)$ followed by ferrous $(10.04 \%)$ and potassium $(6.12 \%)$. The cigarette had calcium and potassium as major components represent $(60.86 \%)$ and $(31.39 \%)$, respectively. Using the GC-MS technique, toxic material of crude material of cigarette and incense was found that cigarette had 60 chemicals and industrial incense had 33 chemicals. Glycerol, 3TMS derivative, and dimethyl phthalate recorded the most dominant chemicals in cigarettes by $19.64 \%$ and $16.75 \%$, respectively. Dimethyl phthalate was the most dominant chemical (75.68\%) in industrial incense. Most cigarette components were recorded as toxic materials while those in the incense had a low toxicity.
\end{abstract}

\section{INTRODUCTION}

Mosquitoes are the world's most severe public health concern spreading a variety of diseases, such as filariasis, avian malaria, Japanese encephalitis, dengue, etc., and causing countless deaths every year (Das et al., 2007). Frequent use of artificial insecticides for mosquito control led to the growth of resistance, adverse effects on nontarget species, and environmental and human health problems have also contributed to search for alternative control measures (Brown, 1986; Hayes and Laws, 1991).

Naturally occurring substances of plant origin with insecticidal qualities have recently been evaluated for the control of numbers of insect pests and vectors. For example, oils of leaf and bark from Cryptomeria japonica displayed high larvicide efficacy toward Aedes aegypti (Diptera: Culicidae) larvae (Cheng et al., 2003). 
Azadiractin which is the active ingredient from neem plant and was recognized for its larvicide mosquito capacity (Alouani et al., 2009). The extracts of Oriandrum sativam, Murraya koenigii, Trigonella foenum graceum and Ferula asafetida had been found to be successful agents against Ae. aegypti and Culex mosquito larvae (Isman 1999, Harve and Kamath 2004;). Phytochemicals extracted from plant origin exhibited larvicide, insect growth regulator, oviposition attractant and repellent (Babu and Murugan 1998; Venkatachalam and Jebanesan 2001a; 2001b).

The tobacco plant (Nicotiana tabacum) contains a high amount of alkaloid which has an insecticidal effect and for decades, people use its mixture with water as a homemade remedy to control garden insects (Booker et al., 2010). The potential larvicidal effects of $N$. tabacum plant extracts against $C x$. quinquefasciatus were evaluated (Ullah et al., 2018). Also, repellent activities of its bio-oil on mosquitoes were reported (Jufri et al., 2016). Cigarette smoke is commonly known as an air pollutant and has had severe side effects on public health. Recently, cigarette smoke has been associated with the severity of coronavirus disease 2019 (Guo, 2020). Whenever industrial incense is burned, it emits smoke containing pollutants and gas products as well as other organic molecules. The fire from burned incense was distinguished as $\mathrm{CO}$, $\mathrm{CO}_{2}, \mathrm{NO}_{2}, \mathrm{SO}_{2}$, and many others that will be emitted to the surrounding environment (Lin et al., 2008).

Popular synthetic bug sprays used for mosquitoes such as DEET, N,N diethyl-mtoluamide that was approved for use as a pesticide, but the potential side effects including, eyes and skin irritation, insomnia and the promotion of angiogenesis which increases tumor growth had also been reported (Legeay et al., 2016). Permethrin, resmethrin, and sumithrin, as well as other industrial pyrethroids that are widely used in mosquito control systems to combat adult mosquitoes showed side effect in altering the nerve function by changing the biochemistry of nerve membrane of sodium channels (Paul et al. 2005; EPA 2009; Trivedi et al., 2018).

Thus, The authors speculated that the long-term exposure to cigarette smoke and industrial incense on $C x$. Pipiens would have a negative effect on the yield at various life stages, which might be related to the toxic substances emitted from both of them. Consequently, the current study aimed to investigate the chemical-related abnormality in the numbers of yielded stages of $C x$. pipiens upon the long-term exposure to smokes of cigarette and industrial incense for two generations.

\section{MATERIALS AND METHODS}

\section{Insect rearing}

Laboratory colonies of $C x$. pipiens were established by collecting egg patches from drain wells situated in Qena Campus of South Valley University, Egypt. Mosquito specimens were reared and identified by following the key of Harbach (1985). In the laboratory, hatched larvae were kept in glass jars $(50 \times 100 \mathrm{~mm})$ with a sufficient amount 
of water. Larvae were provided with finely ground wheat bread crumbs and the jars were covered with fine white mesh allowing ventilation. For all jars, water, and food were replaced daily with clean water and food for the mosquito larvae. Adults were kept in cages under laboratory conditions $25 \pm 5{ }^{\circ} \mathrm{C}, 25 \pm 10 \%$ humidity a photoperiod of $16 \mathrm{~L}: 8 \mathrm{D}$ at $10 \mathrm{~lx}$.

\section{Smokes effect}

The smoke effect resulted from the burning of industrial incense and cigarettes lasted continuously through all life stages for two generations of $C x$. pipiens from February 2019 to May 2019. It was accomplished in isolated chambers $4 \mathrm{~m} \times 2 \mathrm{~m} \times 3 \mathrm{~m}$ in length, width, and height, respectively. Tests were conducted daily by burning $1.5 \mathrm{~g}$ of cigarette materials and $3.5 \mathrm{~g}$ of industrial incense for each treatment chamber. The burning process of cigarette and incense stick lasted for $15 \mathrm{~min}$, while insects exposed to the emitted smoke for about $12 \mathrm{~h}$ inside each chamber. The same protocol was applied daily starting from the eggs stage to the adult stage for two constitutive generations which extended to 82 days. Data were recorded every day of $C x$. pipiens various stages.

\section{XRF analysis}

Crude materials of cigarette and commercial incense were subjected to X-ray fluorescence spectrometer analysis at the Central Laboratory of South Valley University, Qena, Egypt. Internal instrument calibration was performed prior to the sample study. Cigarette and incense contents were examined for $300 \mathrm{~s}$ through a small plastic cup filled with SDI mylar film to evaluate the main and trace elements (Ene et al., 2010; Mccomb et al., 2014).

\section{Sample derivatizations and GC-MS analysis}

Sample derivatization and GC-MS analysis were done by taking $30 \mathrm{mg}$ from each sample which was extracted by $6 \mathrm{ml}$ ethyl acetate following by $3 \mathrm{ml}$ diethyl ether to characterize polar and non-polar components. The organic phase from each extraction was pooled and dried in liquid nitrogen. The dried extract was derivatized with bistrimethylsily ltriflouroacetamide; BSTF 100 ul, trimethylchlorosilane; TMCS 20 ul and pyridine $20 \mathrm{ul}$ at $70{ }^{\circ} \mathrm{C}$ for $30 \mathrm{~min}$ (Villas-Bôas et al., 2006). After cooling the samples were processed for GC-MS analysis. The GC-MS Agilent Technologies device was equipped with gas chromatograph 7890B and mass spectrometer detector 5977A at Central Laboratory Network, National Research Centre, Cairo, Egypt. The GC was packed with HP-5MS column $30 \mathrm{~m} \times 0.25 \mathrm{~mm}$ internal diameter and $0.25 \mu \mathrm{m}$ film thickness. Analyses were accomplished using helium as the carrier gas at $1.0 \mathrm{ml} / \mathrm{min}$ flow rate, at a split 50:1, injection volume of $0.5 \mu \mathrm{l}$ and the following temperature program: 50 ${ }^{\circ} \mathrm{C}$ for one min; rising at $8{ }^{\circ} \mathrm{C} / \mathrm{min}$ to $300{ }^{\circ} \mathrm{C}$ and held for $20 \mathrm{~min}$. The detector and injector were held at $250{ }^{\circ} \mathrm{C}$. Mass spectra were obtained by electron ionization; EI at 70 $\mathrm{eV}$; using a spectral range of $\mathrm{m} / \mathrm{z}$ 30-700 and solvent delay $8 \mathrm{~min}$. The mass temperature was $230{ }^{\circ} \mathrm{C}$ and Quad $150{ }^{\circ} \mathrm{C}$. The identification of different constituents was determined 
by matching the spectrum fragmentation pattern with those stored in NIST Mass Spectral Library data.

\section{Statistical analysis}

Results were subjected to one-way variance analysis; ANOVA, $P<0.05$ was considered to be significant, and mean values were measured using the Least Significant Difference (LSD) using post hoc test.

\section{RESULTS}

\section{Fresh air and stages of $C x$. pipiens}

Data through the two successive generations for numbers of eggs, live larvae, larval moulting and mortality, live pupae, live adults and mortality of $C x$. pipiens under the condition of fresh air, cigarette, and incense smokes are shown in (Table 1). Results showed that under fresh air a very low loss rate has been observed at all stages. Generally, it was found that in the $1^{\text {st }}$ generation there was no adult mortality while at the $2^{\text {nd }}$ generation some adults had been lost. First generation of $C x$. pipiensin each stage to the next showed zero loss rates except larval moulting and mortality. In the second generation, the data revealed that live larvae, larval mortality, pupal number, and live adults showed a variable loss rate. It had also been shown that the different rates observed for live larvae, molting of larval, larval mortality, live pupa and live adults were varied between the two generations in the range between 9.52 and $12.02 \%$.

\section{Cigarette smoke and stages of $C x$. pipiens}

It was evident that cigarette smoke caused loss rates for live larvae, larval mortality, live pupae, live adults, and adult mortality, respectively. In the second generation, cigarette smoke caused a loss in live larvae, larval mortality, pupal numbers, live adults and mortality. Numbers of eggs hatching, live larvae, larval molting, larval mortality, emerged live adults, and adults mortality were found to be in the range between 3.41 and $75.68 \%$. Exposure to cigarette smoke significantly caused disorder in most stages of $C x$. pipiens, whereas the difference rate between $2^{\text {nd }}$ and $1^{\text {st }}$ generations of hatched eggs under the cigarette smoke was found to be $88.06 \%$, and larval mortality $96.39 \%$ and the other stages in the range between $38.62 \%$ and $70.26 \%$ (Table 1). 
Table 1. Impact of smokes from cigarette and industrial incense on the yield numbers of various stages of $C x$. Pipiensfor two generations

\begin{tabular}{|c|c|c|c|c|c|c|c|c|}
\hline $\begin{array}{l}\text { Treat- } \\
\text { ment }\end{array}$ & Generation & Eggs & Live larvae & $\begin{array}{c}\text { Larval } \\
\text { moulting }\end{array}$ & $\begin{array}{c}\text { Larval } \\
\text { mortality }\end{array}$ & Pupal No. & Live adults & $\begin{array}{c}\text { Adult } \\
\text { mortality }\end{array}$ \\
\hline \multirow{3}{*}{$\stackrel{\varrho}{\stackrel{0}{0}}$} & $\operatorname{Iose} \%$ & $\begin{array}{l}7.09 \\
(291)\end{array}$ & $\begin{array}{c}7.09 \\
(291 \pm 9.61)\end{array}$ & $\begin{array}{c}6.15 \\
(252 \pm 2.46)\end{array}$ & $\begin{array}{c}0.46 \\
(19 \pm 0.44)\end{array}$ & $\begin{array}{c}5.68 \\
(233 \pm 2.16)\end{array}$ & $\begin{array}{c}5.68 \\
(233 \pm 2.46)\end{array}$ & 0.00 \\
\hline & $\begin{array}{c}2^{\text {nd }} \\
\text { Loss } \%\end{array}$ & $\begin{array}{c}6.76 \\
(277)\end{array}$ & $\begin{array}{c}6.39 \\
(262 \pm 10.41) \\
5.42 \%\end{array}$ & $\begin{array}{c}13.40 \% \\
5.51 \\
(226 \pm 3.21) \\
13.74 \%\end{array}$ & $\begin{array}{c}0.51 \\
(21 \pm 0.75) \\
8.02 \%\end{array}$ & $\begin{array}{c}5.00 \\
(205 \pm 3) \\
10.24 \%\end{array}$ & $\begin{array}{c}5.00 \\
(205 \pm 3.21) \\
0.00 \%\end{array}$ & $\begin{array}{c}0.71 \\
(29 \pm 1) \\
14.15 \%\end{array}$ \\
\hline & Df $1^{\text {st }} / 2^{\text {nd }}$ & $4.81 \%$ & $9.97 \%$ & $10.32 \%$ & $9.52 \%$ & $12.02 \%$ & $12.02 \%$ & $1.00 \%$ \\
\hline \multirow{3}{*}{ 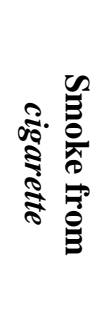 } & Loss $\%$ & $\begin{array}{l}17.98 \\
(737)\end{array}$ & $\begin{array}{c}13.59 \\
(557 \pm 43.85) \\
24.42 \%\end{array}$ & $\begin{array}{c}25.29 \\
(1037 \pm 9.54) \\
46.29 \%\end{array}$ & $\begin{array}{c}4.05 \\
(166 \pm 3.67) \\
29.80 \%\end{array}$ & $\begin{array}{c}9.54 \\
(391 \pm 3.67) \\
27.09 \%\end{array}$ & $\begin{array}{c}4.76 \\
(195 \pm 2.65) \\
50.13 \%\end{array}$ & $\begin{array}{c}2.76 \\
(113 \pm 2.84) \\
57.95 \%\end{array}$ \\
\hline & $\begin{array}{c}2^{\text {nd }} \\
\text { Loss } \%\end{array}$ & $\begin{array}{l}2.15 \\
(88)\end{array}$ & $\begin{array}{c}2.07 \\
\left(85 \pm 5.41^{* *}\right) \\
3.41 \%\end{array}$ & $\begin{array}{c}2.01 \\
(86 \pm 4.40 * *) \\
1.16 \%\end{array}$ & $\begin{array}{c}0.15 \\
(6 \pm 0.36 * *) \\
7.06 \%\end{array}$ & $\begin{array}{c}2.85 \\
(80 \pm 2.15) \\
6.98 \%\end{array}$ & $\begin{array}{c}1.41 \\
(58 \pm 1.09) \\
27.50 \%\end{array}$ & $\begin{array}{c}1.07 \\
(44 \pm 1.09 * *) \\
75.68 \%\end{array}$ \\
\hline & Df $1^{\text {st }} / 2^{\text {nd }}$ & $88.06 \%$ & $52.05 \%$ & $91.71 \%$ & $96.39 \%$ & $38.62 \%$ & $70.26 \%$ & $61.06 \%$ \\
\hline \multirow{3}{*}{ 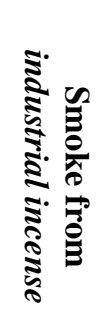 } & $\begin{array}{c}1 \text { st } \\
\text { Loss \% }\end{array}$ & $\begin{array}{l}12.34 \\
(506)\end{array}$ & $\begin{array}{c}17.15 \\
(471 \pm 57.34) \\
6.92 \%\end{array}$ & $\begin{array}{c}24.76 \\
(1015 \pm 23.83) \\
53.59 \%\end{array}$ & $\begin{array}{c}11.68 \\
(247 \pm 15.87) \\
47.56 \%\end{array}$ & $\begin{array}{c}5.46 \\
(224 \pm 3.14) \\
9.31 \%\end{array}$ & $\begin{array}{c}4.49 \\
(184 \pm 2.21) \\
17.86 \%\end{array}$ & $\begin{array}{c}1.46 \\
(60 \pm 1.07) \\
32.61 \%\end{array}$ \\
\hline & $\begin{array}{c}2^{\text {nd }} \\
\text { Loss } \%\end{array}$ & $\begin{array}{l}19.59 \\
(803)\end{array}$ & $\begin{array}{c}2.61 \\
\left(107 \pm 3.06^{* *}\right) \\
86.67 \%\end{array}$ & $\begin{array}{c}1.97 \\
(81 \pm 2.19) \\
24.29 \%\end{array}$ & $\begin{array}{c}1.10 \\
(45 \pm 1.13) \\
42.06 \%\end{array}$ & $\begin{array}{c}1.34 \\
(55 \pm 1.04 *) \\
48.60 \%\end{array}$ & $\begin{array}{c}0.80 \\
(33 \pm 0.89 *) \\
4.00 \%\end{array}$ & $\begin{array}{c}0.44 \\
(18 \pm 0.63) \\
54.55 \%\end{array}$ \\
\hline & $\mathrm{Df} 1^{\mathrm{st}} / 2^{\mathrm{nd}}$ & $36.99 \%$ & $77.28 \%$ & $92.02 \%$ & $81.78 \%$ & $75.45 \%$ & $82.07 \%$ & $70.00 \%$ \\
\hline
\end{tabular}




\section{Industrial incense smokes and stages of $C x$. pipiens}

Results show smoke effect of industrial incense on the two generations of $C x$. pipiens. $1^{\text {st }}$ generation of smoke effect from industrial incense caused loss rate for the larval mortality and adult mortality. The $2^{\text {nd }}$ generation of $C x$. pipiens had been affected by loss rate for live larvae, larval mortality, pupal numbers and adult mortality. Live larvae, larval moulting and mortality, adult mortality and live adults negatively decreased from 86.67 and $4.00 \%$. However, difference in rates between the two generations on eggs showed increasing by $36.99 \%$. That means, egg production of the $1^{\text {st }}$ to $2^{\text {nd }}$ generations was affected negatively by smoke of cigarette and positively by industrial incense (Table 1).

\section{XRF analysis}

Cigarette and industrial incense substances were analyzed for their mineral phases by XRF, which showed variable quantities (Fig. 1, 2). Dominant and non-dominant elements were quantified in cigarette and industrial incense substances. Incense showed a major amount of Calcium followed by Ferrous and Potassium. Silica, Manganese, Zinc, and Titanium were identified to be minor components in the range between 3.32 and $0.68 \%$. Cigarette had Calcium and Potassium as major components representing $60.86 \%$ and $31.39 \%$ respectively. Ferrous, Silica, Manganese, Bromine, Strontium, and Sulfur found to be minor components in the range between 2.96 and $0.10 \%$ (Table 2).

\section{Phytocomponents in cigarette and industrial incense raw materials}

Identification and characterization of the phytocomponents found in the ethyl acetate and diethylether extracts to know polar and nonpolar chemicals present in cigarette and incense are shown (Tables 3 and 4; Figs 2 and 3). Sixty peaks were recognized from the GC-MS chromatogram for the cigarette of which the most common compounds found are Glycerol, 3TMS derivative, followed by Dimethyl phthalate, 5.alpha.-Androstan-1.alpha.-methyl-3.alpha.,17.beta.-diol, di-trimethylsilyl, alpha.Linolenic acid, TMS derivative, Pyridine, 3-(1-methyl-2-pyrrolidinyl)-, (S)-, D-(-)Fructofuranose, pentakis(trimethylsilyl) ether (isomer 2). The remaining fifty three chemicals were found in less amount of in the range between (0.20 to 2.79\%) (Table 3, Fig. 2). Industrial incense had thirty-three peaks and the most dominant chemical is Dimethyl phthalate representing, followed by Methyl trimethylsilyl phthalate. Chemicals found in fewer amounts are Decanoic acid, TBDMS derivative, Phthalic acid, 2TMS derivative, Cyclopentaneacetic acid, 3-oxo-2-pentyl-, methyl ester. Also, there were chemicals found in very less amount in the range between $0.08 \%$ to $0.71 \%$ (Table 4 , Fig. 4).

Table 2. XRF analysis of raw materials of cigarette and industrial incense

\begin{tabular}{lll}
\hline Element \% & Raw materials of cigarette \% & $\begin{array}{l}\text { Raw materials of } \\
\text { industrial incense \% }\end{array}$ \\
\hline $\mathbf{S i}$ & 1.4909 & 3.3248 \\
$\mathbf{K}$ & 31.3946 & 6.1237 \\
$\mathbf{C a}$ & 60.8629 & 78.2430 \\
$\mathbf{M n}$ & 0.6791 & 0.8506 \\
$\mathbf{F e}$ & 2.4062 & 10.0409 \\
$\mathbf{Z n}$ & Not determined & 0.7371 \\
$\mathbf{T i}$ & Not determined & 0.6799 \\
$\mathbf{B r}$ & 0.1083 & Not determined \\
$\mathbf{S r}$ & 0.0985 & Not determined \\
$\mathbf{S}$ & 2.9594 & Not determined \\
\hline
\end{tabular}




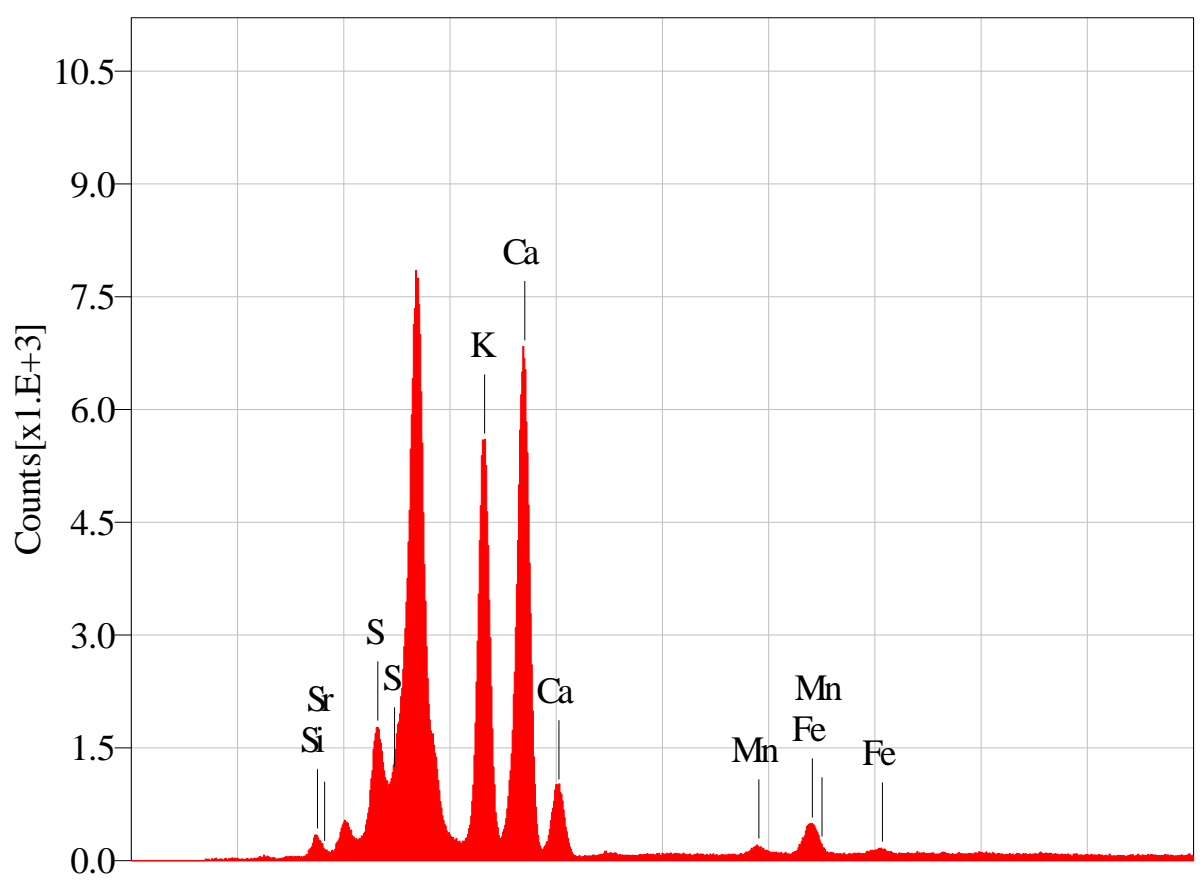

Fig. 1 XRF material output of cigarettes.

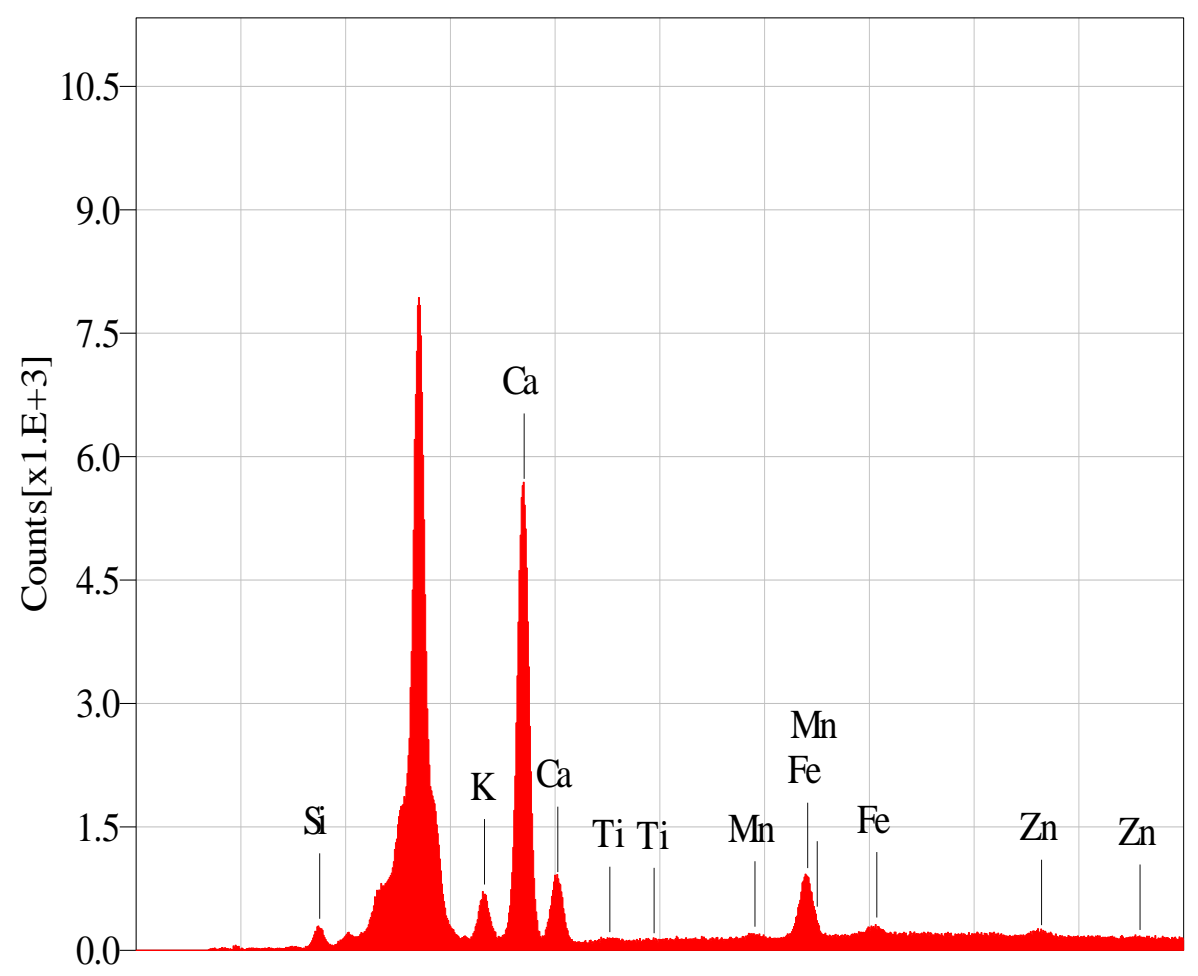

Fig. 2 XRF material output of industrial incense. 


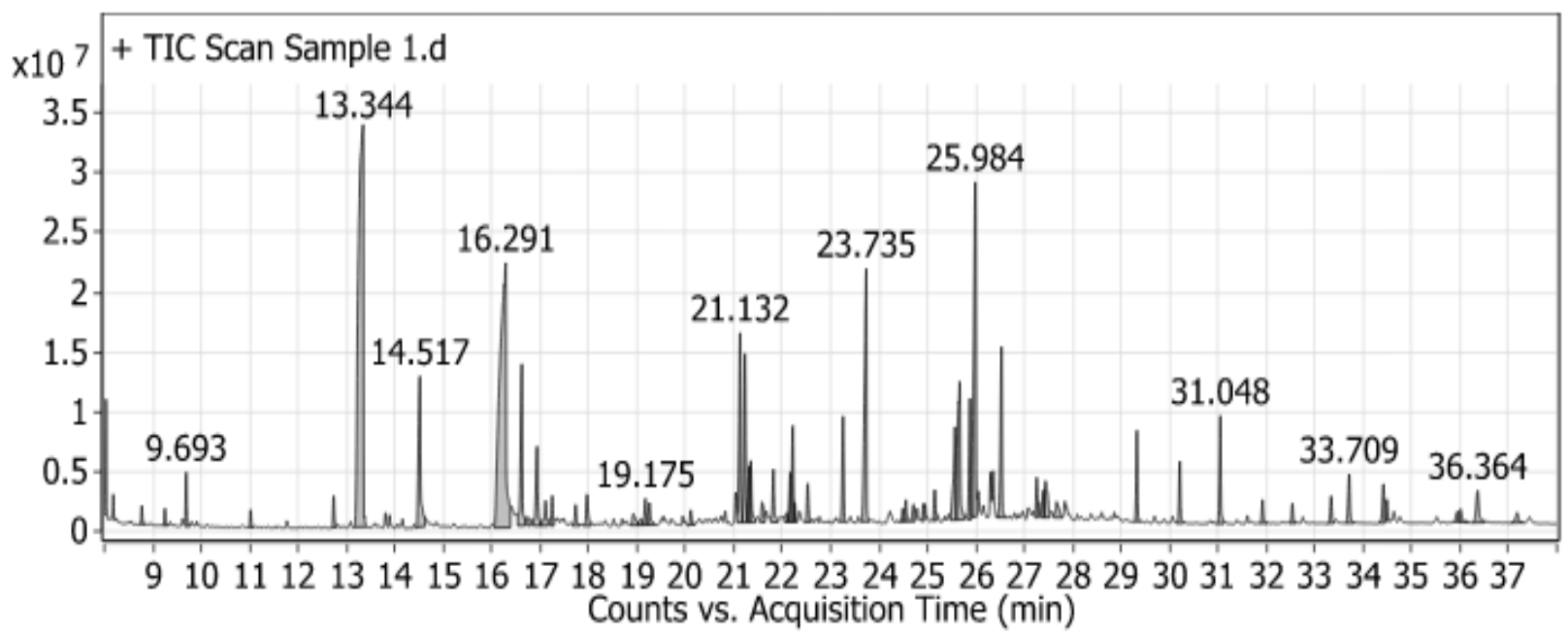

Fig. 3 Typical chromatograph of GC-MS with identified chemical from extract of ethyl acetate and diethyletherof raw material of cigarette.

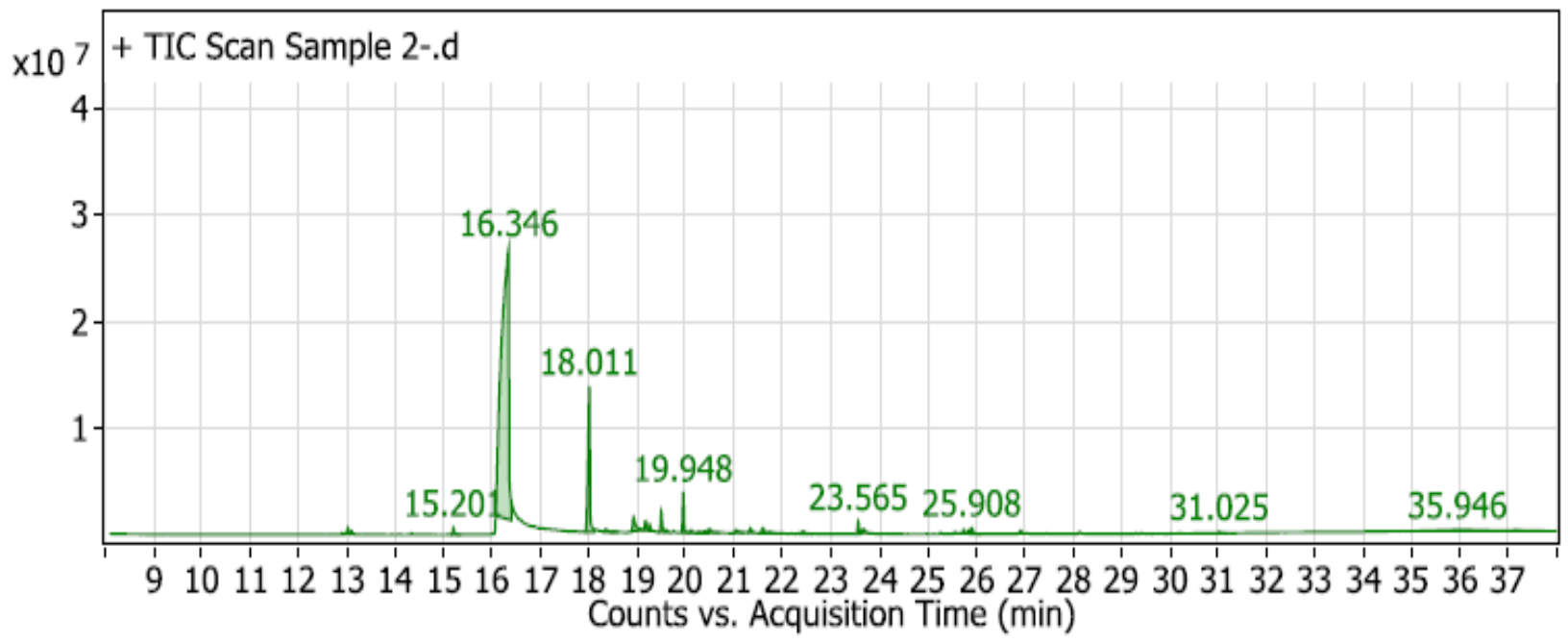

Fig. 4 Typical chromatograph of GC-MS with identified chemical from extract of ethyl acetate and diethylether of raw material of industrial incense.

Table 3. GC-MS analysis of ethyl acetate and diethylether extracts of raw materials of cigarette

\begin{tabular}{|c|c|c|c|c|c|}
\hline Peak & RT & Name & Formula & Area & $\begin{array}{c}\text { Area } \\
\text { Sum } \\
\%\end{array}$ \\
\hline 1 & 8.772 & Propylene glycol, 2TMS derivative & $\mathrm{C}_{9} \mathrm{H}_{24} \mathrm{O}_{2} \mathrm{Si}_{2}$ & 2774659.7 & 0.23 \\
\hline 2 & 9.253 & Tris(trimethylsilyl)carbamate & $\mathrm{C}_{10} \mathrm{H}_{27} \mathrm{NO}_{2} \mathrm{Si}_{3}$ & 2518988.3 & 0.21 \\
\hline 3 & 9.693 & D-(-)-Lactic acid, 2TMS derivative & $\mathrm{C}_{9} \mathrm{H}_{22} \mathrm{O}_{3} \mathrm{Si}_{2}$ & 7642719.1 & 0.63 \\
\hline 4 & 11.021 & Hydracrylic acid, 2TMS derivative & $\mathrm{C}_{9} \mathrm{H}_{22} \mathrm{O}_{3} \mathrm{Si}_{2}$ & 2492883.8 & 0.21 \\
\hline 5 & 12.737 & Benzoic Acid, TMS derivative & $\mathrm{C}_{10} \mathrm{H}_{14} \mathrm{O}_{2} \mathrm{Si}$ & 5485902 & 0.45 \\
\hline 6 & 13.344 & Glycerol, 3TMS derivative & $\mathrm{C}_{12} \mathrm{H}_{32} \mathrm{O}_{3} \mathrm{Si}_{3}$ & 237671251 & 19.64 \\
\hline 7 & 14.517 & Pyridine, 3-(1-methyl-2-pyrrolidinyl)-, (S)- & $\mathrm{C}_{10} \mathrm{H}_{14} \mathrm{~N}_{2}$ & 40498040 & 3.35 \\
\hline 8 & 16.291 & Dimethyl phthalate & $\mathrm{C}_{10} \mathrm{H}_{10} \mathrm{O}_{4}$ & 202674517 & 16.75 \\
\hline
\end{tabular}




\begin{tabular}{|c|c|c|c|c|c|}
\hline 9 & 16.623 & Malic acid, 3TMS derivative & $\mathrm{C}_{13} \mathrm{H}_{30} \mathrm{O}_{5} \mathrm{Si}_{3}$ & 34482905 & 2.8 \\
\hline 10 & 16.76 & $\begin{array}{l}\text { 1-Benzenesulfonohydrazide, } \mathrm{N}^{\prime}- \\
\text { tricyclo[4.2.2.0(1,5)dec-8-ylidene }\end{array}$ & $\mathrm{C}_{16} \mathrm{H}_{20} \mathrm{~N}_{2} \mathrm{O}_{2} \mathrm{~S}$ & 2514927.4 & 0.21 \\
\hline 11 & 16.937 & 2'-Deoxyribolactone, 2 TMS derivative & $\mathrm{C}_{11} \mathrm{H}_{24} \mathrm{O}_{4} \mathrm{Si}_{2}$ & 18584116 & 1.5 \\
\hline 12 & 17.115 & L-5-Oxoproline, , 2TMS derivative & $\mathrm{C}_{11} \mathrm{H}_{23} \mathrm{NO}_{3} \mathrm{Si}_{2}$ & 7083156.5 & 0.5 \\
\hline 13 & 17.252 & 3-Aminothiophenol, 2TMS derivative & $\mathrm{C}_{12} \mathrm{H}_{23} \mathrm{NSSi}_{2}$ & 5649964.2 & $0.4^{4}$ \\
\hline 14 & 17.733 & $\begin{array}{l}\text { L-Threonic acid, tris(trimethylsilyl) ether, } \\
\text { trimethylsilyl ester }\end{array}$ & $\mathrm{C}_{16} \mathrm{H}_{40} \mathrm{O}_{5} \mathrm{Si}_{4}$ & 4697027.2 & 0.3 \\
\hline 15 & 17.967 & Monomethyl phthalate, TMS derivative & $\mathrm{C}_{12} \mathrm{H}_{16} \mathrm{O}_{4} \mathrm{Si}$ & 8001740.7 & 0.66 \\
\hline 16 & 18.923 & $\begin{array}{l}\alpha \text {-d-Xylopyranoside, methyl-2,3,4-tris-O- } \\
\text { [9-borabicyclo[3.3.1]non-9-yl]- }\end{array}$ & $\mathrm{C}_{30} \mathrm{H}_{51} \mathrm{~B}_{3} \mathrm{O}_{5}$ & 4567488.8 & 0.38 \\
\hline 17 & 19.095 & 10,12-Tricosadiynoic acid, TMS derivative & $\mathrm{C}_{26} \mathrm{H}_{46} \mathrm{O}_{2} \mathrm{Si}$ & 2382974.6 & 0.2 \\
\hline 18 & 19.175 & $\begin{array}{l}\text { 4-(3,3-Dimethyl-but-1-ynyl)-4-hydroxy- } \\
\text { 2,6,6-trimethylcyclohex-2-enone }\end{array}$ & $\mathrm{C}_{15} \mathrm{H}_{22} \mathrm{O}_{2}$ & 6213514.5 & 0.51 \\
\hline 19 & 19.255 & $\begin{array}{l}\text { Spiro[tricyclo[4.4.0.0(5,9)]decane-10,2'- } \\
\text { oxirane], 1-methyl-4-isopropyl-7,8-dihy }\end{array}$ & $\mathrm{C}_{15} \mathrm{H}_{24} \mathrm{O}_{3}$ & 5618365.7 & 0.4 \\
\hline 20 & 20.113 & $\begin{array}{l}\text { 3-(2-Hydroxyethyl)phenol, TBDMS } \\
\text { derivative }\end{array}$ & $\mathrm{C}_{14} \mathrm{H}_{24} \mathrm{O}_{2} \mathrm{Si}$ & 3176540.8 & 0.2 \\
\hline 21 & 21.132 & $\begin{array}{l}\text { D-(-)-Fructofuranose, } \\
\text { pentakis(trimethylsilyl) ether (isomer 2) }\end{array}$ & $\mathrm{C}_{21} \mathrm{H}_{52} \mathrm{O}_{6} \mathrm{Si}_{5}$ & 38794419 & 3.2 \\
\hline 22 & 21.229 & $\begin{array}{l}\text { D-Psicofuranose, pentakis(trimethylsilyl) } \\
\text { ether (isomer 2) }\end{array}$ & $\mathrm{C}_{21} \mathrm{H}_{52} \mathrm{O}_{6} \mathrm{Si}_{5}$ & 35250604 & 2.9 \\
\hline 23 & 21.32 & $\begin{array}{l}\text { D-(-)-Fructopyranose, } 5 \text { TMS derivative } \\
\text { (isomer 1) }\end{array}$ & $\mathrm{C}_{21} \mathrm{H}_{52} \mathrm{O}_{6} \mathrm{Si}_{5}$ & 10173939 & 0.8 \\
\hline 24 & 21.349 & Myristic acid, TMS derivative & $\mathrm{C}_{17} \mathrm{H}_{36} \mathrm{O}_{2} \mathrm{Si}$ & 12179520 & 1.01 \\
\hline 25 & 21.584 & 1,5-Anhydrohexitol, 4TMS derivative & $\mathrm{C}_{18} \mathrm{H}_{44} \mathrm{O}_{5} \mathrm{Si}_{4}$ & 6040132.5 & 0.5 \\
\hline 26 & 21.818 & Quininic acid (5TMS) & $\mathrm{C}_{22} \mathrm{H}_{52} \mathrm{O}_{6} \mathrm{Si}_{5}$ & 11007784 & 0.91 \\
\hline 27 & 22.093 & D-Fructose, 5TMS derivative & $\mathrm{C}_{21} \mathrm{H}_{52} \mathrm{O}_{6} \mathrm{Si}_{5}$ & 2449105.6 & 0.2 \\
\hline 28 & 22.167 & D-(-)-Tagatose, 5TMS derivative & $\mathrm{C}_{21} \mathrm{H}_{52} \mathrm{O}_{6} \mathrm{Si}_{5}$ & 9901431.5 & 0.82 \\
\hline 29 & 22.219 & D-Glucose, 5TMS derivative & $\mathrm{C}_{21} \mathrm{H}_{52} \mathrm{O}_{6} \mathrm{Si}_{5}$ & 17073798 & 1.41 \\
\hline 30 & 22.259 & L-(-)-Sorbose, 5TMS derivative & $\mathrm{C}_{21} \mathrm{H}_{52} \mathrm{O}_{6} \mathrm{Si}_{5}$ & 2888303.1 & 0.24 \\
\hline 31 & 22.528 & $\begin{array}{l}\text { Acrylic acid, 2,3-bis[(trimethylsilyl)oxy]-, } \\
\text { trimethylsilyl ester }\end{array}$ & $\mathrm{C}_{12} \mathrm{H}_{28} \mathrm{O}_{4} \mathrm{Si}_{3}$ & 7443298.9 & 0.62 \\
\hline 32 & 23.255 & D-Glucopyranose, 5TMS derivative & $\mathrm{C}_{21} \mathrm{H}_{52} \mathrm{O}_{6} \mathrm{Si}_{5}$ & 19075624 & 1.58 \\
\hline 33 & 23.735 & Palmitic Acid, TMS derivative & $\mathrm{C}_{19} \mathrm{H}_{40} \mathrm{O}_{2} \mathrm{Si}$ & 61244523 & 5.0 \\
\hline 34 & 24.49 & Doconexent, TMS derivative & $\mathrm{C}_{25} \mathrm{H}_{40} \mathrm{O}_{2} \mathrm{Si}$ & 2622457.6 & 0.2 \\
\hline 35 & 24.553 & Myo-Inositol, 6TMS derivative & $\mathrm{C}_{24} \mathrm{H}_{60} \mathrm{O}_{6} \mathrm{Si}_{6}$ & 4411643.9 & 0.36 \\
\hline 36 & 24.708 & Doconexent, TBDMS derivative & $\mathrm{C}_{28} \mathrm{H}_{46} \mathrm{O}_{2} \mathrm{Si}$ & 4092456.1 & 0.34 \\
\hline 37 & 24.92 & Epitestosterone, TMS derivative & $\mathrm{C}_{22} \mathrm{H}_{36} \mathrm{O}_{2} \mathrm{Si}$ & 3392316.7 & 0.28 \\
\hline 38 & 25.149 & Epimethendiol-diOTMS & $\mathrm{C}_{26} \mathrm{H}_{48} \mathrm{O}_{2} \mathrm{Si}_{2}$ & 6376744.2 & 0.5 \\
\hline 39 & 25.566 & $\begin{array}{l}\text { 9,12-Octadecadienoic acid (Z,Z)-, TMS } \\
\text { derivative }\end{array}$ & $\mathrm{C}_{21} \mathrm{H}_{40} \mathrm{O}_{2} \mathrm{Si}$ & 25318828 & 2.05 \\
\hline 40 & 25.664 & .alpha.-Linolenic acid, TMS derivative & $\mathrm{C}_{21} \mathrm{H}_{38} \mathrm{O}_{2} \mathrm{Si}$ & 44963501 & 3.72 \\
\hline 41 & 25.875 & Stearic acid, TMS derivative & $\mathrm{C}_{21} \mathrm{H}_{44} \mathrm{O}_{2} \mathrm{Si}$ & 22453372 & 1.86 \\
\hline 42 & 25.984 & $\begin{array}{l}\text { 5.alpha.-Androstan-1.alpha.-methyl- } \\
\text { 3.alpha.,17.beta.-diol, di-trimethylsilyl }\end{array}$ & $\mathrm{C}_{26} \mathrm{H}_{50} \mathrm{O}_{2} \mathrm{Si}_{2}$ & 85638137 & 7.08 \\
\hline 43 & 26.528 & Epitestosterone, TMS derivative & $\mathrm{C}_{22} \mathrm{H}_{36} \mathrm{O}_{2} \mathrm{Si}$ & 33788945 & 2.79 \\
\hline 44 & 27.254 & Eicosapentaenoic Acid, TBDMS derivative & $\mathrm{C}_{26} \mathrm{H}_{44} \mathrm{O}_{2} \mathrm{Si}$ & 7652859.9 & 0.63 \\
\hline 45 & 27.397 & 5,8,11-Eicosatriynoic acid, TMS derivative & $\mathrm{C}_{23} \mathrm{H}_{36} \mathrm{O}_{2} \mathrm{Si}$ & 5988980.6 & 0.49 \\
\hline 46 & 27.437 & $\begin{array}{l}\text { 11-Hydroxyetiocholanolone } \\
\text { (3.alpha.,5.alpha.,11.beta.)-, 2TMS } \\
\text { derivative }\end{array}$ & $\mathrm{C}_{25} \mathrm{H}_{46} \mathrm{O}_{3} \mathrm{Si}_{2}$ & 13234467 & 1.09 \\
\hline
\end{tabular}




\begin{tabular}{llllll}
$\mathbf{4 7}$ & 27.666 & $\begin{array}{l}\text { 5,8,11-Eicosatriynoic acid, tert- } \\
\text { butyldimethylsilyl ester }\end{array}$ & $\mathrm{C}_{26} \mathrm{H}_{42} \mathrm{O}_{2} \mathrm{Si}$ & 6614646.3 & 0.55 \\
$\mathbf{4 8}$ & 29.326 & 1-Monopalmitin, 2TMS derivative & $\mathrm{C}_{25} \mathrm{H}_{54} \mathrm{O}_{4} \mathrm{Si}_{2}$ & 14119055 & 1.17 \\
$\mathbf{4 9}$ & 30.213 & Sucrose, 8TMS derivative & $\mathrm{C}_{36} \mathrm{H}_{86} \mathrm{O}_{11} \mathrm{Si}_{8}$ & 11206862 & 0.93 \\
$\mathbf{5 0}$ & 31.048 & Glycerol monostearate, 2TMS derivative & $\mathrm{C}_{27} \mathrm{H}_{58} \mathrm{O}_{4} \mathrm{Si}_{2}$ & 18603304 & 1.54 \\
$\mathbf{5 1}$ & 31.918 & Nonacosane & $\mathrm{C}_{29} \mathrm{H}_{60}$ & 4444265.7 & 0.37 \\
$\mathbf{5 2}$ & 32.536 & Octadecane, 3-ethyl-5-(2-ethylbutyl)- & $\mathrm{C}_{26} \mathrm{H}_{54}$ & 3725700.5 & 0.31 \\
$\mathbf{5 3}$ & 33.337 & Heptacosane & $\mathrm{C}_{27} \mathrm{H}_{56}$ & 5611588.9 & 0.46 \\
$\mathbf{5 4}$ & 33.709 & Hentriacontane & $\mathrm{C}_{31} \mathrm{H}_{64}$ & 11036525 & 0.91 \\
$\mathbf{5 5}$ & 34.418 & .alpha.-Tocopherol, TMS derivative & $\mathrm{C}_{32} \mathrm{H}_{58} \mathrm{O}_{2} \mathrm{Si}$ & 9393527.6 & 0.78 \\
$\mathbf{5 6}$ & 34.487 & Triacontane, 1-bromo- & $\mathrm{C}_{30} \mathrm{H}_{61} \mathrm{Br}$ & 4826384.4 & 0.4 \\
$\mathbf{5 7}$ & 35.94 & Campesterol, TMS derivative & $\mathrm{C}_{31} \mathrm{H}_{56} \mathrm{OSi}$ & 3198913 & 0.26 \\
$\mathbf{5 8}$ & 36.009 & Cholest-22-ene-21-ol, 3,5-dehydro-6- & $\mathrm{C}_{33} \mathrm{H}_{54} \mathrm{O}_{3}$ & 4193104 & 0.35 \\
& & methoxy-, pivalate & & & \\
$\mathbf{5 9}$ & 36.364 & Stigmasterol, TMS derivative & $\mathrm{C}_{32} \mathrm{H}_{56} \mathrm{OSi}$ & 11207903 & 0.93 \\
$\mathbf{6 0}$ & 37.176 & .beta.-Sitosterol, TMS derivative & $\mathrm{C}_{32} \mathrm{H}_{58} \mathrm{OSi}$ & 3783643.3 & 0.31 \\
\hline
\end{tabular}

Table 4. GC-MS analysis of ethyl acetate and diethylether extracts of raw materials of industrial incense

\begin{tabular}{|c|c|c|c|c|c|}
\hline & RT & Name & Formula & Area & $\begin{array}{c}\text { Area } \\
\text { Sum \% }\end{array}$ \\
\hline 1 & 13.023 & $\begin{array}{l}\text { Tripropylene glycol monomethyl ether, } \\
\text { TMS derivative }\end{array}$ & $\mathrm{C}_{13} \mathrm{H}_{30} \mathrm{O}_{4} \mathrm{Si}$ & 1480969.2 & 0.41 \\
\hline 2 & 15.201 & .beta.-Eudesmol, TMS derivative & $\mathrm{C}_{18} \mathrm{H}_{34} \mathrm{OSi}$ & 1433694.7 & 0.39 \\
\hline 3 & 16.346 & Dimethyl phthalate & $\mathrm{C}_{10} \mathrm{H}_{10} \mathrm{O}_{4}$ & 275222001 & 75.68 \\
\hline 4 & 18.011 & Methyl trimethylsilyl phthalate & $\mathrm{C}_{12} \mathrm{H}_{16} \mathrm{O}_{4} \mathrm{Si}$ & 39369759 & 10.83 \\
\hline 5 & 18.35 & $\begin{array}{l}\text { 2-Propenal, 3-(2,6,6-trimethyl-1- } \\
\text { cyclohexen-1-yl)- }\end{array}$ & $\mathrm{C}_{12} \mathrm{H}_{18} \mathrm{O}$ & 1166609.7 & 0.32 \\
\hline 6 & 18.524 & $\begin{array}{l}\text { 1-Benzenesulfonohydrazide, } \mathrm{N}^{\prime}- \\
\text { tricyclo[4.2.2.0(1,5)dec-8-ylidene }\end{array}$ & $\mathrm{C}_{16} \mathrm{H}_{20} \mathrm{~N}_{2} \mathrm{O}_{2} \mathrm{~S}$ & 303696.12 & 0.08 \\
\hline 7 & 18.923 & $\begin{array}{l}\text { Cyclopentaneacetic acid, 3-oxo-2-pentyl-, } \\
\text { methyl ester }\end{array}$ & $\mathrm{C}_{13} \mathrm{H}_{22} \mathrm{O}_{3}$ & 5456772.7 & 1.5 \\
\hline 8 & 19.172 & $\begin{array}{l}\text { 4-(3,3-Dimethyl-but-1-ynyl)-4-hydroxy- } \\
\text { 2,6,6-trimethylcyclohex-2-enone }\end{array}$ & $\mathrm{C}_{15} \mathrm{H}_{22} \mathrm{O}_{2}$ & 2088256.6 & 0.57 \\
\hline 9 & 19.255 & $\begin{array}{l}\text { 4-(3,3-Dimethyl-but-1-ynyl)-4-hydroxy- } \\
\text { 2,6,6-trimethylcyclohex-2-enone }\end{array}$ & $\mathrm{C}_{15} \mathrm{H}_{22} \mathrm{O}_{2}$ & 1247646.6 & 0.34 \\
\hline 10 & 19.496 & Phthalic acid, 2TMS derivative & $\mathrm{C}_{14} \mathrm{H}_{22} \mathrm{O}_{4} \mathrm{Si}_{2}$ & 4133074.5 & 1.14 \\
\hline 11 & 19.594 & 2,5-Octadecadiynoic acid, methyl ester & $\mathrm{C}_{19} \mathrm{H}_{30} \mathrm{O}_{2}$ & 656475.49 & 0.18 \\
\hline 12 & 19.948 & Decanoic acid, TBDMS derivative & $\mathrm{C}_{16} \mathrm{H}_{34} \mathrm{O}_{2} \mathrm{Si}$ & 7102461.5 & 1.95 \\
\hline 13 & 20.106 & $\begin{array}{l}\text { Benzoic acid, 2-methoxy-4-methyl-3-nitro-, } \\
\text { methyl ester }\end{array}$ & $\mathrm{C}_{10} \mathrm{H}_{11} \mathrm{NO}_{5}$ & 643245.73 & 0.18 \\
\hline 14 & 20.28 & Falcarinol & $\mathrm{C}_{17} \mathrm{H}_{24} \mathrm{O}$ & 763529.81 & 0.21 \\
\hline 15 & 20.468 & 2,5-Octadecadiynoic acid, methyl ester & $\mathrm{C}_{19} \mathrm{H}_{30} \mathrm{O}_{2}$ & 893127.04 & 0.25 \\
\hline 16 & 20.506 & Doconexent & $\mathrm{C}_{22} \mathrm{H}_{32} \mathrm{O}_{2}$ & 1130214 & 0.31 \\
\hline 17 & 21.041 & $\begin{array}{l}\text { 3H-Cyclodeca[b]furan-2-one, 4,9- } \\
\text { dihydroxy-6-methyl-3,10-dimethylene- } \\
\text { 3a,4,7,8,9,1 }\end{array}$ & $\mathrm{C}_{15} \mathrm{H}_{20} \mathrm{O}_{4}$ & 1207428.4 & 0.33 \\
\hline 18 & 21.334 & $\begin{array}{l}\text { 5,8,11-Eicosatrienoic acid, (Z)-, TMS } \\
\text { derivative }\end{array}$ & $\mathrm{C}_{23} \mathrm{H}_{42} \mathrm{O}_{2} \mathrm{Si}$ & 1058153.2 & 0.29 \\
\hline 19 & 21.598 & 7-Acetyl-6-ethyl-1,1,4,4-tetramethyltetralin & $\mathrm{C}_{18} \mathrm{H}_{26} \mathrm{O}$ & 1605190 & 0.44 \\
\hline 20 & 21.734 & 3.beta.,17.beta.-dihydroxyestr-4-ene & $\mathrm{C}_{18} \mathrm{H}_{28} \mathrm{O}_{2}$ & 582729.56 & 0.16 \\
\hline
\end{tabular}




\begin{tabular}{|c|c|c|c|c|c|}
\hline 21 & 22.435 & $\begin{array}{l}\text { 5,8,11,14-Eicosatetraenoic acid, methyl } \\
\text { ester, (all-Z)- }\end{array}$ & $\mathrm{C}_{21} \mathrm{H}_{34} \mathrm{O}_{2}$ & 1111454.5 & 0.31 \\
\hline 22 & 23.565 & Ethylene brassylate & $\mathrm{C}_{15} \mathrm{H}_{26} \mathrm{O}_{4}$ & 2586271.1 & 0.71 \\
\hline 23 & 23.67 & 2,4-Difluorobenzene, 1-benzyloxy- & $\mathrm{C}_{13} \mathrm{H}_{10} \mathrm{~F}_{2} \mathrm{O}$ & 1027027.8 & 0.28 \\
\hline 24 & 25.652 & $\begin{array}{l}\text { 7,10-Epoxytricyclo[4.2.1.1(2,5)]decane, } 1 \text { - } \\
\text { trimethylsilyl- }\end{array}$ & $\mathrm{C}_{13} \mathrm{H}_{22} \mathrm{OSi}$ & 322667.04 & 0.09 \\
\hline 25 & 25.75 & $\begin{array}{l}\text { 9,12-Octadecadiynoic acid, trimethylsilyl } \\
\text { ester }\end{array}$ & $\mathrm{C}_{21} \mathrm{H}_{36} \mathrm{O}_{2} \mathrm{Si}$ & 1000782.7 & 0.28 \\
\hline 26 & 25.848 & 2,5-Octadecadiynoic acid, methyl ester & $\mathrm{C}_{19} \mathrm{H}_{30} \mathrm{O}_{2}$ & 811544.86 & 0.22 \\
\hline 27 & 25.908 & $\begin{array}{l}\text { Bicyclo[5.3.0]decan-2-one, 9- } \\
\text { trimethylsilylmethylene }\end{array}$ & $\mathrm{C}_{14} \mathrm{H}_{24} \mathrm{OSi}$ & 1939257.5 & 0.53 \\
\hline 28 & 26.926 & $\begin{array}{l}\text { 9,12,15-Octadecatrienoic acid, } 2 \text { - } \\
\text { [(trimethylsilyl)oxy }]-1- \\
\text { [[(trimethylsilyl)oxy }] \mathrm{m}\end{array}$ & $\mathrm{C}_{27} \mathrm{H}_{52} \mathrm{O}_{4} \mathrm{Si}_{2}$ & 1140693.6 & 0.31 \\
\hline 29 & 31.025 & $\begin{array}{l}\text { t-Butyl-(2-[3-(2,2-dimethyl-6-methylene- } \\
\text { cyclohexyl)-propyl]-[1,3]dithian-2-yl)- } \\
\text { dimethyl-silane }\end{array}$ & $\mathrm{C}_{22} \mathrm{H}_{42} \mathrm{~S}_{2} \mathrm{Si}$ & 384914.45 & 0.11 \\
\hline 30 & 35.946 & $\begin{array}{l}\text { Androstane-11,17-dione, 3- } \\
\text { [(trimethylsilyl)oxy]-, 17-[O- } \\
\text { (phenylmethyl)oxime], (3.alpha.,5.alpha.)- }\end{array}$ & $\mathrm{C}_{29} \mathrm{H}_{43} \mathrm{NO}_{3} \mathrm{Si}$ & 2581664.8 & 0.71 \\
\hline 31 & 37.151 & Cyclobarbital & $\mathrm{C}_{12} \mathrm{H}_{16} \mathrm{~N}_{2} \mathrm{O}_{3}$ & 301337.68 & 0.08 \\
\hline 32 & 45.094 & $\begin{array}{l}\text { 9,12,15-Octadecatrienoic acid, 2- } \\
\text { [(trimethylsilyl)oxy }]-1- \\
\text { [[(trimethylsilyl)oxy]m }\end{array}$ & $\mathrm{C}_{27} \mathrm{H}_{52} \mathrm{O}_{4} \mathrm{Si}_{2}$ & 2578951 & 0.71 \\
\hline 33 & 50.165 & 1,4-Bis(trimethylsilyl) benzene & $\mathrm{C}_{12} \mathrm{H}_{22} \mathrm{Si}_{2}$ & 337876.47 & 0.09 \\
\hline
\end{tabular}

\section{DISCUSSION}

To the best of our knowledge, no studies have been published about the effect of the long-term toxic effect of inhaling cigarette and industrial incense smokes on mosquitos. Results of the current study indicated that mosquito biology behaviors were more negatively affected by cigarette smoke than industrial smokes. Effects of cigarette smoke on the first and the second generations of Cx. pipiensexhibited loss rates towards the hatched numbers of eggs, alive larvae, larval moulting and mortality, alive pupae, alive adults, and adult mortality more than industrial incense. This may be due to the differences between cigarette and incense chemical components as indicated from GCMS and XRF results. Regardless of the lack of overall differences between the impact of cigarette and industrial incense smokes, there was some interesting complexity in the results. For instance, under cigarette smoke, eggs in the $1^{\text {st }}$ generation were seen to be not affected. However, under incense smoke, the number of eggs increased. These results may be explained by the nature of the insect egg shell which may be less affected with smoke or gases from industrial incense smoke/or it causes mutation that allows adult female to lay an increased numbers of eggs (need more investigations). It has been verified that the egg stage of the insect is difficult to monitor due to its unusual egg shell structure, which consists of several layers to allow the embryo to breathe industrial incense smoke, and previously has been shown to be as a barrier to insecticides (Campbell et al., 2016). In addition, the sensitivity of how the egg shell hardens varies depending on the age of the egg, as the hardness changes over time, and the embryo may develop more enzymes that break down insecticides (Jacobs et al., 2013). 
The negative impact of cigarette smoke may be from the genetic mutation on various stages of $C x$. pipiens. Many other mutation patterns associated with smoking (Blackford et al., 2009).

The susceptibility level of mosquito's stages to cigarette and industrial incense smokes through the two generations was different from each other. It was found that the effect of incense smokes on the live larvae produced a loss rate that was higher compared with the effect of cigarette smoke. The metal component of incense should be taken into consideration. It was proved by XRF analysis the presence of $\mathrm{Zn}$ but not in cigarette which was reported to be used in different forms to manage mosquito larvae (Mostafa $\boldsymbol{e t}$ al., 2018). The differences in the loss rate of the live larvae, larval moulting and mortality, live pupae, pupal mortality, and adult mortality through the exposure to smokes for two constitutive generations may have occurred because of the possible morphological malformation.

Regarding GC-MS analysis of cigarette, there were sixty peaks, some of these peaks referred to toxic chemicals found such as D-(-)-Lactic acid, 2TMS derivative, Pyridine, 3-(1-methyl-2-pyrrolidinyl)-, (S)-, Dimethyl phthalate and 3-Aminothiophenol, 2TMS derivative, which was reported as good insect repellent (Brown and Hebert, 1997; Jantan and Zaki, 1998).

\section{CONCLUSION}

This is the first demonstration of cigarette and industrial incense smokes directly inhaled by $C x$. pipiens for continuous 82 days, which is important to investigate the alteration in the physiological response associated with smoking to other organisms. Our works suggested that long-term inhaling of the smoke from cigarette continuously could alter all successive stages of $C x$. pipiens more severely than the industrial incense smoke. This is probably related to the toxicity of the chemicals as revealed by GC-MS and XRF analysis. Our results may be beneficial for further exploration of the toxicological mechanism of identified chemicals from cigarette and incense smokes against $C x$. pipiens and human.

\section{ACKNOWLEDGMENT}

We would like to show our gratitude to Dr. Margo Mire, Lincoln, University of Missouri, for her valuable and insightful comments reviewing an early version of this article.

\section{REFERENCES}

Alouani, A.; Rehimi, N. and Soltani, N. (2009). Larvicidal activity of a neem tree extract (Azadirachtin) against mosquito larvae in the Republic of Algeria. Jordan J. Biol. Sci.,2: 15-22. 
Babu, R. and Murugan, K. (1998). Interactive effect of neem seed kernel and neem gum extracts on the control of Culex quinquefasciatus Say. Neem Newsletter, 15: 911.

Blackford, A.; Parmigiani, G.; Kensler, T.W.; Wolfgang, C.; Jones, S.; Zhang, X.; Parsons, D. W.; Lin, J. C. H.; Leary, R. J. and Eshleman, J. R. (2009). Genetic mutations associated with cigarette smoking in pancreatic cancer. Cancer Res.,69 (6): 3681-3688.10.1158/0008-5472.CAN-09-0015

Booker, C. J.; Bedmutha, R.;Vogel, T.; Gloor, A.; Xu, R.; Ferrante, L.; Yeung, K. K. C.; Scott, I. M.; Conn, K. L. and Berruti, F. (2010). Experimental investigations into the insecticidal, fungicidal, and bactericidal properties of pyrolysis bio-oil from tobacco leaves using a fluidized bed pilot plant. Ind. Eng. Chem. Res.,49(20): 10074-10079.https://doi.org/10.1021/ie100329z

Brown, A. (1986). Insecticide resistance in mosquitoes: a pragmatic review. J. Am. Mosq. Control Assoc.2 (2): 123-140.

Brown, M. and Herbert, A. A. (1997). Insect repellents: an overview. J. Am. Acad. Dermatol., 36: 243-249. 10.1016/s0190-9622(97)70289-5

Campbell, B. E.; Pereira, R. M. and Koehler, P. G. (2016). Complications with controlling insect eggs. Insecticide Resistance; Intech: Rijeka, Croatia: 83-96. http:://dx.doi.org/10.5772/61848

Cheng, S. S.,Chang, H. T.,Chang, S. T.,Tsai, K. H. and Chen, W. J. (2003). Bioactivity of selected plant essential oils against the yellow fever mosquito Aedes aegypti larvae. Bioresour. Technol., 89(1): 99-102. https://doi.org/10.1016/S09608524(03)00008-7

Das, N., Goswami, D. and Rabha, B. (2007). Preliminary evaluation of mosquito larvicidal efficacy of plant extracts. J. Vector Borne Dis., 44: 145.

Ene, A.,Bosneaga, A. and Georgescu, L. (2010). Determination of heavy metals in soils using XRF technique. Rom. Journ. Phys.,55: 815-820.

EPA, (2009). Reregistration Eligibility Decision (RED) for Permethrin. 738-R-06-017.

Guo, F.R. (2020). Active smoking is associated with severity of coronavirus disease 2019 (CVID-19): An update of a meta-analysis. Tob. Induc. Dis., 18. https://doi.org/1O0.18332/tid/121915.

Harbach, R.E. (1985). Pictorial keys to the genera of mosquitoes, subgenera of Culex and the species of Culex (Culex) occurring in southwestern Asia and Egypt, with a 
note on the subgeneric placement of Culex deserticola (Diptera: Culicidae). Walter Reed Army Inst Of Research Washington DC.17 (2): 83-107

Harve, G. and Kamath, V. (2004). Larvicidal activity of plant extracts used alone and in combination with known synthetic larvicidal agents against Aedes aegypti. Indian J. Exp. Biol., 42 (12): 1216-1219.

Hayes, W. J. and Laws, E .R. (1991). Handbook of pesticide toxicology.(1): 1897-1908

Isman, M. (1999). Pesticides based on plant essential oils-these oils and their terpenoidal constituents could have a role in pest and disease management. Pestic. Outlook, 10: 68-72.

Jacobs, C. G., Rezende, G. L., Lamers, G. E. and Van Der Zee, M. (2013). The extraembryonic serosa protects the insect egg against desiccation. Proceedings of the Royal Society B: Biological Sciences, 280: 20131082.

Jantan, I. and Zaki, Z. M. (1998). Development of environment-friendly insect repellents from the leaf oils of selected Malaysian plants. ASEAN Review of Biodiversity and Environmental Conservafion (ARBEC), 6: 1-7.

Jufri, M.; Irmayanti, E. and Gozan, M. (2016). Formulation of tobacco based mosquito repellent to avoid dengue fever. Int. J. Pharm.Tech. Res.,9: 140-145.

Legeay, S.;Clere, N.; Hiiairet, G.; DO, Q.T.; Bernard, P.,Quignard, J.F.; ApaireMarchais, V.; Lapied, B. and Faure, S. (2016). The insect repellent N, N-diethyl-mtoluamide (DEET) induces angiogenesis via allosteric modulation of the M3 muscarinic receptor in endothelial cells. Sci. rep., 6: 28546.

Lin, T. C.; Krishnasw Amy, G.; and Chi, D. S. (2008).Incense smoke: clinical, structural and molecular effects on airway disease. Clin. Mol. Allergy., 6: 3.10.1186/1476-7961-6-3

Mccomb, J. Q.; Rogers, C.; Han, F. X. and Tchounwou, P. B. (2014). Rapid screening of heavy metals and trace elements in environmental samples using portable X-ray fluorescence spectrometer, A comparative study. Water Air Soil Pollut.,225: 2169.10.1007/s11270-014-2169-5

Mostafa, W. A.; Elgazzar, E., Beall; G.W.,Rashed, S.S. and Rashed, E.M. (2018). Insecticidal effect of zinc oxide and aluminum oxide nanoparticles synthesized by co-precipitation technique on Culex quinquefasciatus larvae (Diptera: Culicidae). Int. J. Appl. Res., 4: 290-297. 
Paul, E.; Simonin, H.; and Tomajer, T. (2005). A comparison of the toxicity of synergized and technical formulations of permethrin, sumithrin, and resmethrin to trout. Arch. Environ. Contam. Toxicol., 48(2): 251-259.10.1007/s00244-003-0110-9

Trivedi, A.; Rai, P.; Kumar, J. and Trivedi, C.A.(2018). Formulation of low smoke herbal mosquito repellent sticks by using different essential oils. The Pharma Innovation Journal,7: 173-175.175.

Uliah, Z.; Ijaz, A.; Mughal, T.K. and Zia, K. (2018). Larvicidal activity of medicinal plant extracts against Culex quinquefasciatus Say (Culicidae, Diptera). Int J Mosq Res5: 47-51.

Venkatachalam, M.; and Jebanesan, A. (2001a). Larvicidal activity of Hydrocotyle javanica Thunb.(Apiaceae) extract against Culex quinquefasciatus. J. Exp.Zool. India, 4: 99-101.

Venkatachalam, M.; and Jebanesan, A. (2001b). Repellent activity of Ferronia elephantum Corr.(Rutaceae) leaf extract against Aedes aegypti (L.). Bioresour. Technol.,76: 287-288.

Villas-Boas, S. G.; Noel, S.; Lane, G. A.; Attwood, G. and Cookson, A. (2006). Extracellular metabolomics: a metabolic footprinting approach to assess fiber degradation in complex media. Anal. Biochem., 349: 297-305. 